\title{
Modeling Electric Field and Potential Distribution of an Model of Insulator in Two Dimensions by the Finite Element Method
}

\author{
Nassima M'ziou ${ }^{1,2}$, Hani Benguesmia ${ }^{1,3^{*}}$, Hilal Rahali ${ }^{3}$ \\ ${ }^{1}$ Laboratoire de Recherche (LI3CUB), Biskra University, ALGERIA \\ ${ }^{2}$ Department of Electrical Engineering, Faculty of engineer Sciences, Boumerdes University, ALGERIA \\ ${ }^{3}$ Department of Electrical Engineering, Faculty of Technology, M'sila University, B.P. 166, 28000 M'sila, ALGERIA \\ E-mail*: hanibenguesmia16@gmail.com
}

\begin{abstract}
The electrical effects can be written by two magnitudes the field and the electrostatic potential, for the determination of the distribution of the field and the electric potential along the leakage distance of the polluted insulator, the comsol multiphysics software based on the finite element method will be used. The objective of this paper is the modeling electric field and potential distribution in Two Dimensions by the Finite Element Method on a model of insulator simulating the $1512 \mathrm{~L}$ outdoor insulator used by the Algerian company of electricity and gas (SONELGAZ). This model is under different conductivity, applied voltage, position of clean layer and width of clean layer. The computer simulations are carried out by using the COMSOL multiphysics software. This paper describes how Comsol Multiphysics have been used for modeling of the insulator using electrostatic $2 D$ simulations in the AC/DC module. Numerical results showed a good agreement.
\end{abstract}

Keywords: Model of insulator, Pollution, High voltage, Potential distribution, Eectric field.

Received: 30/05/2018 - Accepted: 25/06/2018

\section{Introduction}

The pollution flashover, observed on insulators used in high voltage transmission, is one of the most important problems for power transmission. It is a very complex problem due to several factors such as the modeling difficulties of complex shapes of the insulators, different pollution density at different regions, non-homogeneous pollution distribution on the insulator surface and unknown effect of humidity on the pollution. In the literature, some static and dynamic models were developed by making some assumptions and omissions to predict the flashover voltages of polluted insulators $[1,2]$.

The problematic related to such a study, is in the difficulty to determine, experimentally, in a precise way, the distribution of the potential and moreover, that of the electric field along polluted insulators. Indeed, these measurements, which can only be performed in the laboratory, require heavy and sophisticated equipment, usually expensive [3].

However, there is an alternative to experimental measurements, consisting of the use of digital tools to determine electrical potential and field distributions along polluted insulators. With the increasing development of increasingly powerful computing and numerical computation software, it is now possible to obtain fast and accurate results.

It is very interesting to have a simulation environment that includes the possibility of adding different physical phenomena to the studied model. It is in this philosophy that Comsol Multiphysics has been developed. It is a finite element numerical computation software allowing to model a large variety of physical phenomena characterizing a real problem.

Among the numerical methods available and applicable to electromagnetic calculations, the Finite Element Method. Thus, its use, using commercial software COMSOL Multiphysics, was used to carry out the various simulations [4]. It is very interesting to have a simulation environment that includes the possibility of adding different physical phenomena to the studied model. It is in this philosophy that Comsol Multiphysics has been developed.

The determination of the distribution of the field and the electric potential on the insulating surfaces, in the presence of the pollution, has been the subject of numerous researches [5-7].

The main aim of our work is to see the distribution of the field and electric potential on a type 1512L cap and 
pin insulator model of artificially polluted high voltage, under AC voltage, subjected to various parameters such as the conductivities.

\section{Description of the insulator model}

In our simulation, we chose model $1512 \mathrm{~L}$ insulator plan. The dimensions of the model defined by Moula et Hilal [8,9], The model is a plane that is constituted by a glass plate of $(500 \times 500 \mathrm{~mm})$ on which are placed two electrodes, one of ground and the other of high voltage, and the two electrodes are placed on two aluminum conductive bands that are cut $(500 \mathrm{x} 500 \mathrm{~mm})$ for the glass and $(500 \times 30 \mathrm{~mm})$ for the two electrodes. The distance between two electrodes is $292 \mathrm{~mm}$.

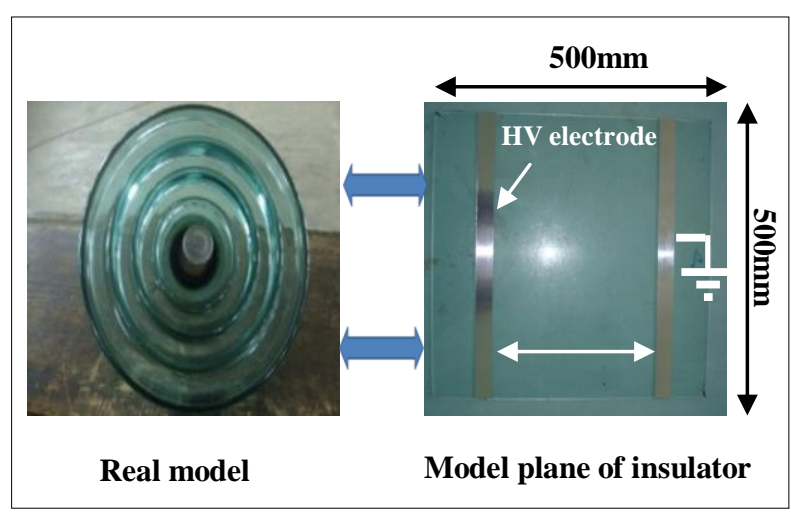

Figure 1. Real model vs Model plane of insulator.

\section{Identification of domains in the comsol software}

COMSOL Multiphysics is an analysis software and finite element solver, which has various applications in physics and engineering especially coupled phenomena, or multi-physics. The different domains of the insulator studied in COMSOL Multi-physics were defined by introducing for each of them the dielectric constant $\varepsilon_{\mathrm{r}}$ and the conductivity $\sigma(\mu \mathrm{s} / \mathrm{cm})$.

The model is a plane that consists of three parts with different properties: the glass having a relative permittivity equal to $\varepsilon_{\mathrm{r}}=6$, and the conductivity $\sigma=10^{-12}$, the aluminum electrodes of relative permittivity of $\varepsilon_{\mathrm{r}}=10^{6}$ and conductivity of $\sigma=10^{6}$, attached to the polluted layers having a relative permittivity of 80 and of which we vary the conductivity. The air a rounding the insulator has a permittivity of $\varepsilon_{\mathrm{r}}=1$ and $\sigma=10^{-14}$.

This software uses the finite element method; the simulation of the model of insulator can be summarized in the following four successive steps:

- The first step is to define the two-dimensional geometry of the model in comsol.

- The second step is the definition of the electrical properties of the materials present. This consists in defining the relative permittivities and the volumic conductivities for each part of the model. In addition, it is necessary to define the boundary conditions that result in the potential imposed on each electrode (Dirichlet conditions).

- The third step is devoted to solving the problem by applying the numerical method and by the construction of the system of equations $(-\operatorname{div} \varepsilon \overrightarrow{\operatorname{grad}} V-\operatorname{div} \sigma \overrightarrow{\operatorname{grad}} V=0)$, and this in introducing the factors of each part of the equation.

- The final step is to display the results as the electrical potential and field with their modules, following each part of the plane model and their distributions.

\section{Simulations results}

Several application-specific modules are available for COMSOL Multiphysics. We chose the AC/DC module. This module simulates electrical components and devices that depend on electrostatic, magnetostatic and electromagnetic quasi-static and another application.

For the case studied, we have taken as boundary conditions for the active electrode (HV) a constant potential equal to $30 \mathrm{kV}$ (high voltage) and ground electrode a 1 potential equal to $0 \mathrm{~V}$.

The mesh density of the finite elements is important for critical areas (close to the surface of the insulator) where greater precision is required, because the electrical properties of the materials are very changeable. Indeed, we considered 1376 elements, and after refining we find 5504 elements in the case of a polluted insulator model. the mesh is illustrated in the clean case, in the figure 2.

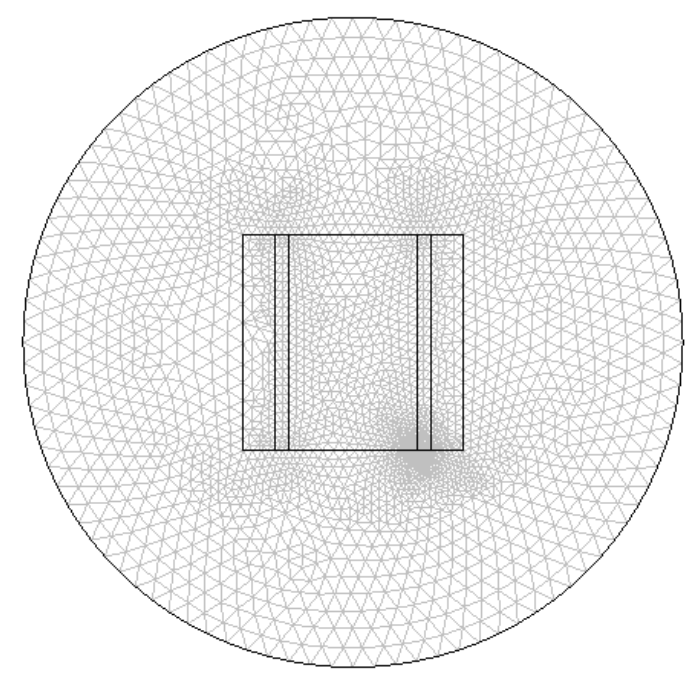

Figure 3. Mesh of the plane model

The study of potential and electric field distributions is along a plane model of real $1512 \mathrm{~L}$ insulator. To illustrate the impact of any parameter denoted $\mathrm{x}$, we plot the potential and the field as a function of the isolator leakage distance for different $\mathrm{L}$, with $\mathrm{L} \Leftrightarrow(\mathrm{L} 1, \mathrm{~L} 2$ and L3) shown in figure 3. 


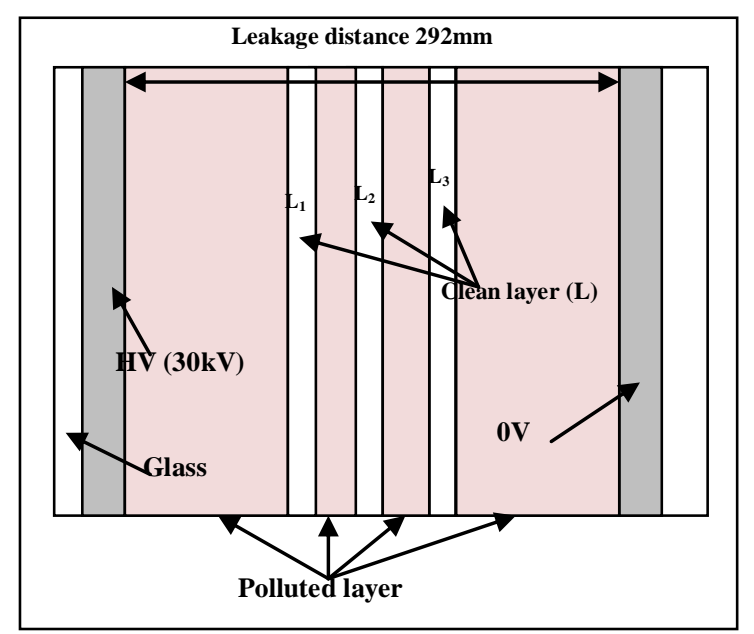

Figure 3. Plan model with all specifications

\section{IV.1. Distribution of electrical potential}

We are interested in determining the potential distribution along the insulator leakage distance as a function of the conductivities. For this, we have introduced in the software different conductivities values (700, 1200, 2400, $3000 \mu \mathrm{S} / \mathrm{cm})$, with the applied voltage equal to $30 \mathrm{kV}$ and a clean layer (Li) fixed, equal to $3 \mathrm{~cm}$.

Figures 4 to 5 illustrates respectively the variation of the potential along of the leakage distance and the potential distribution for the conductivities examined.

There is a low influence on the potential distribution when you vary the values of the conductivities, the potential distribution along the leakage distance of a 1512L model, a rapid reduction in clean layer levels and a slowdown in the polluted layers. If the clean model the potential decreases in a linear way, on the other hand for the polluted case the clean layer ( $\mathrm{Li}$ ) forms an obstacle when the potential stays stable then decreases quickly until the potential equal to $0 \mathrm{~V}$.

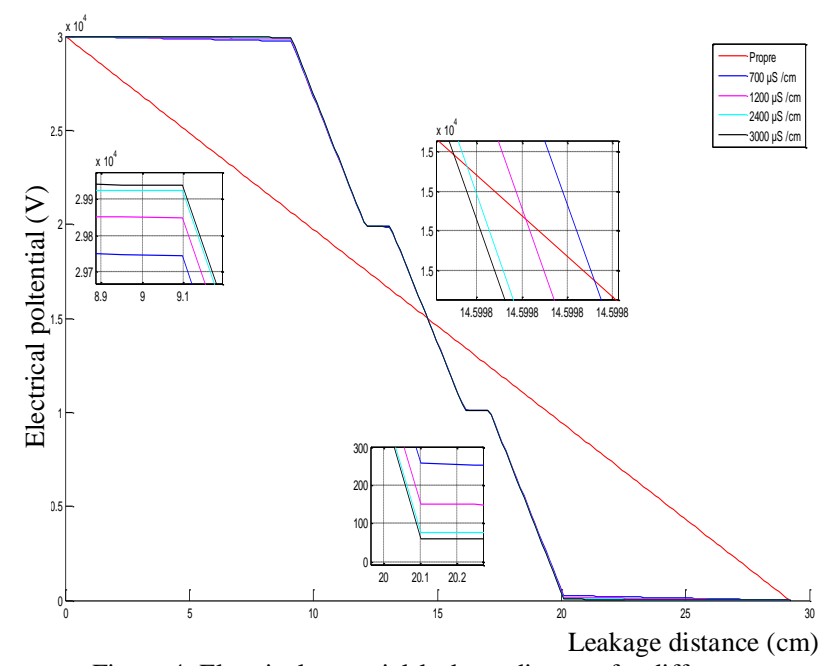

Figure 4. Electrical potential-leakage distance for different conductivities

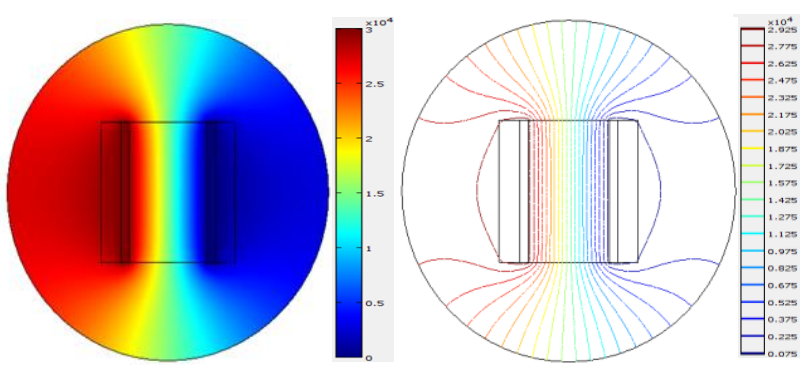

Clean state
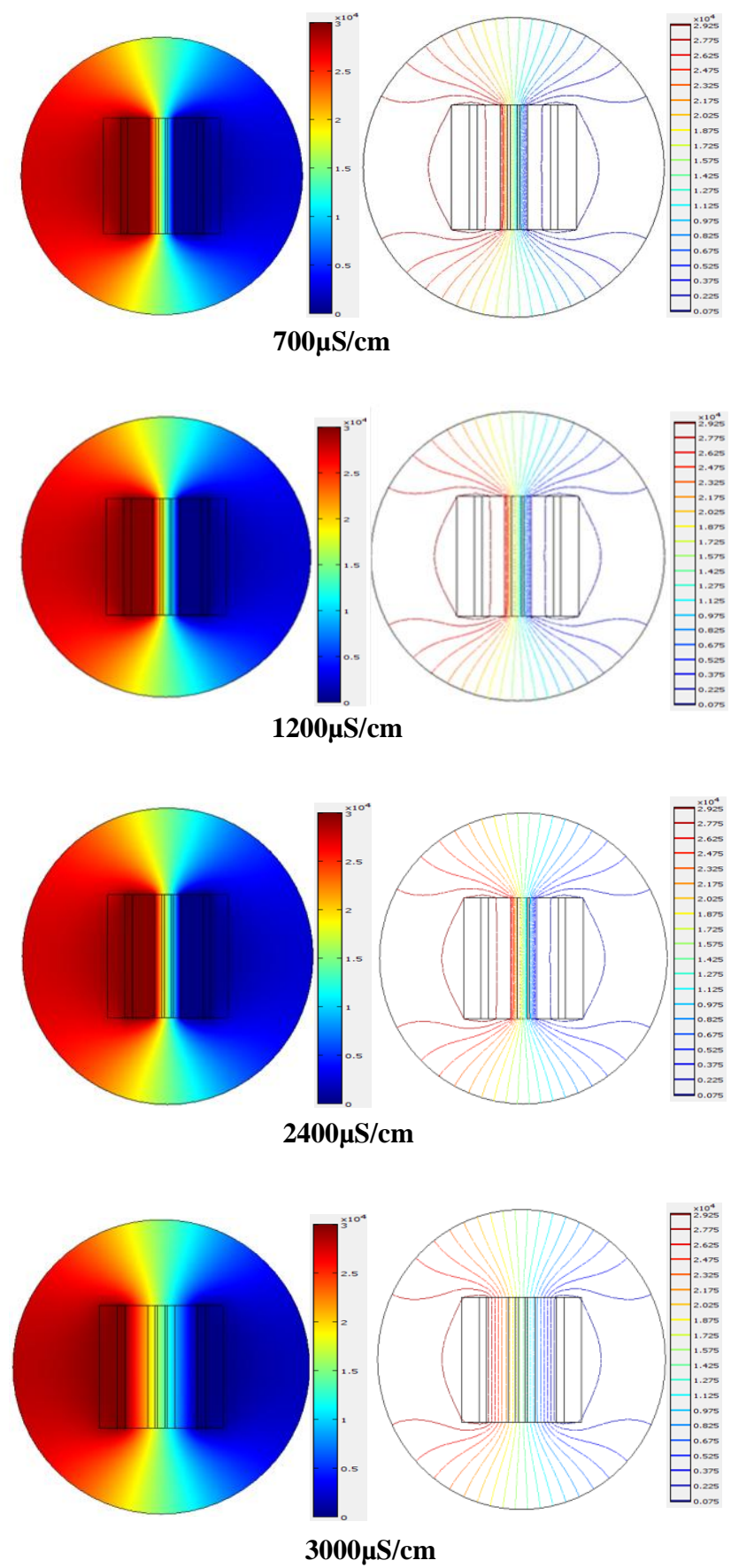

Figure 5. Distribution of electrical potential for various conductivities in two cases, Echogram \& Equipotential lines 


\section{IV.2. Distribution of electrical field}

We are interested in the influence of the conductivity on the electric field distribution for different values of the conductivity $(700,1200,2400,3000 \mu \mathrm{S} / \mathrm{cm})$ with applied voltage equal to $30 \mathrm{kV}$.

Figures 6 to 7 respectively illustrate the variation of the electric field along the leakage distance and the electric field distribution for the conductivities cited above.

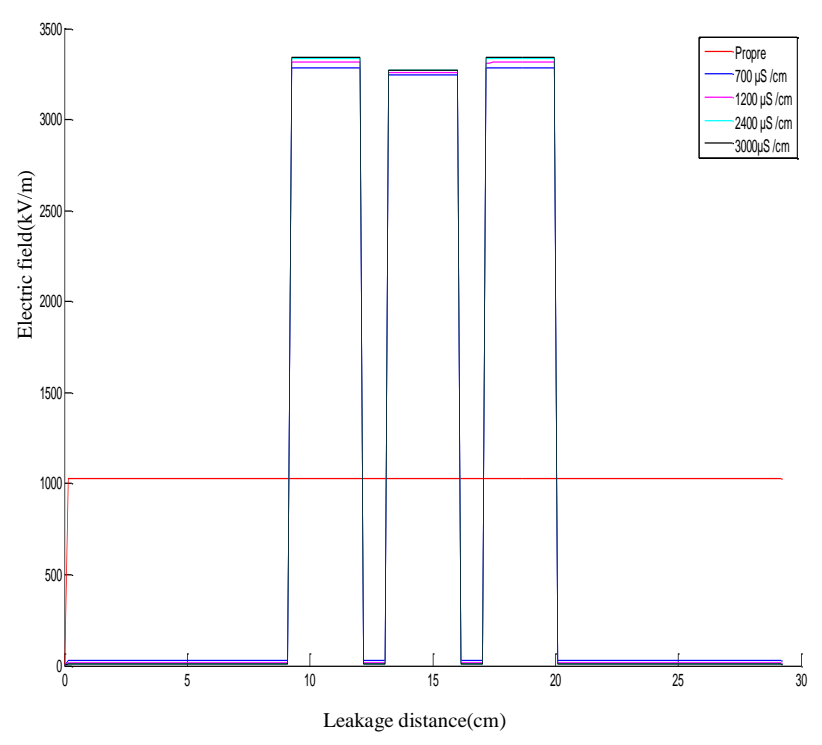

Figure 6. Electric field-leakage distance for different conductivities

We noticed after the appearance of the results that the change of the conductivities has a slight effect on the intensity of the electric field; it is zero inside the two electrodes, because aluminum foil is a perfect conductor. in the proper case the electric field take the value 1000 $\mathrm{kV} / \mathrm{m}$ is remains constant along the leakage distance to the ground electrode is canceled.

In figure 7, you can see for example in the case own the model corners is in red, which explains the intensity of the electric heat is very high, the concentration of electric arcs on the corners of the model, may be due to shape of the model, can also be explained by the movements of concentrated electrons in these corners (leakage current).
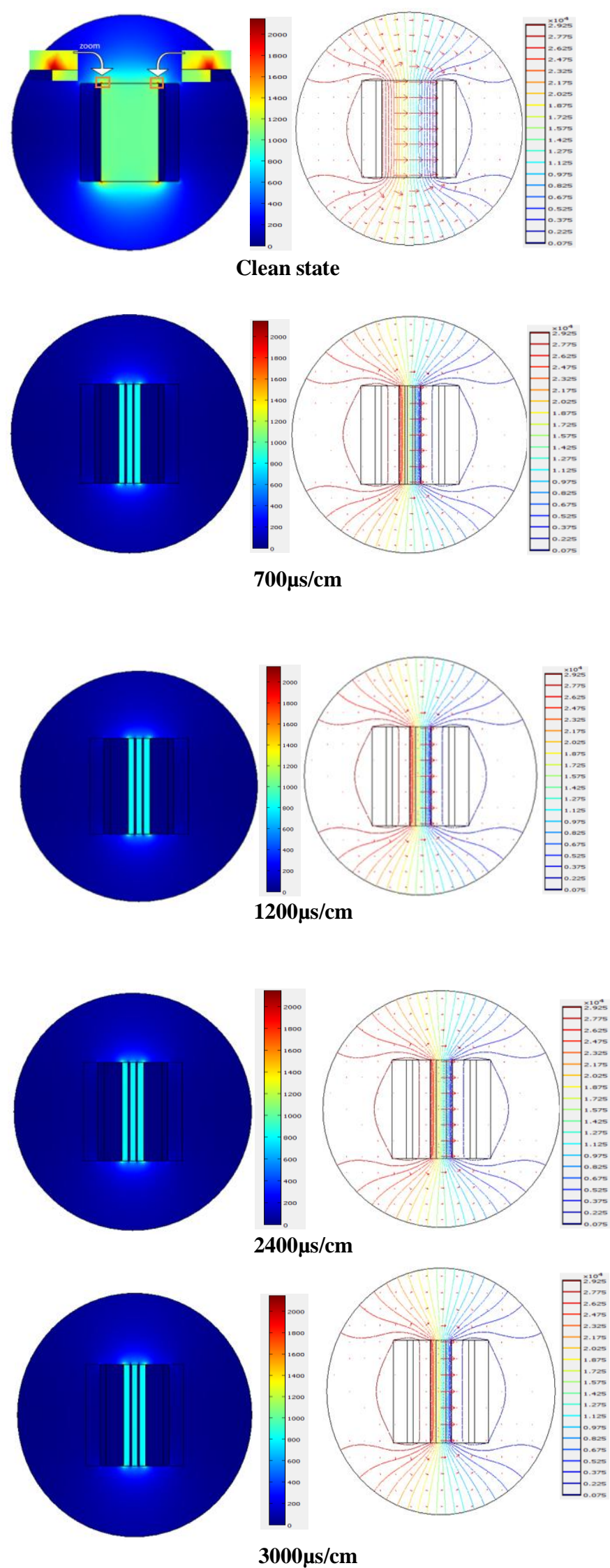

Figure 7. Distribution of electric field for various conductivities in two cases, Echogram \& Equipotential lines 


\section{Conclusion}

In this paper we present the results of different simulations performed on the model of a $1512 \mathrm{~L}$ isolator.

Using the COMSOL Multiphysics software, based on the finite element method, we have been able to analyze the potential and electric field distributions of the $1512 \mathrm{~L}$ plane model, subjected to electro-geometric parameter, namely the conductivities.

As main results, we can say that the conductivities of the polluting layer have practically no effect on the distributions of the potential and the electric field. On the other hand, the intensity of the electric field is more important at the level of the clean layer.

The vector of the electric field in the plan model is parallel because the configuration of the geometry represents a distribution of the uniform electric field. (plan-plan).

The variation of the conductivities has a slight influence on the intensity of the electric field.

\section{Acknowledgements}

We thank the anonymous referees for their useful suggestion.

\section{References}

[1] H. Benguesmia, N. M'ziou, A. Boubakeur, Simulation of The Potential and Electric Field Distribution On High Voltage Insulator Using The Finite Element Method, Diagnostyka, vol 19 (2), 2018, pp 41-52.

[2] H. Benguesmia, N. M'ziou, A.M. Chouchou, L Rachdi, Experimental Study of the various pollution and simulation of potential and electric field distribution using FEMM at a high voltage insulator under alternative current, International Symposium on Computational and Experimental Investigations of Fluid and Structure Dynamics, (CEFSD2015-94), Hammamet, Tunisia, March 16-18, 2015, pp 144. ISBN: 978--9938--9564--2--9.

[3] A. Nekahi, S. G. momeekin, M., Farzaneh, Effect of pollution severity on electric field distribution along a polymeric insulator, In : Properties and Applications of Dielectric Materials (ICPADM), 2015 IEEE 11th International Conference on the. IEEE, 2015, pp 612-615.

[4] COMSOL Multiphysics, User's Manual, Version 5.

[5] T. Zhao, M. G. Comber, Calculation of electric field and potential distribution along non-ceramic insulators considering the effects of conductors and transmission towers, IEEE Transactions on Power Delivery, vol 15 (1), 2000, pp 313-318.

[6] L. A. Dissado, J. C. Fothergill, Electrical degradation and breakdown in polymers, IET (No.9), 1992.

[7] Arshad, A. Nekahi, S.G. McMeekin, M. Farzaneh, Effect of Pollution Severity on Electric Field Distribution along a Polymeric Insulator, 11th International Conference on the
Properties and Applications of Dielectric Materials (ICPADM) 2015. ( in press)

[8] B.Moula, A. Mekhaldi, M. Teguar, Evaluation of insulator's energy under uniform pollution condition, In: Energy Conference and Exhibition (ENERGYCON), 2012 IEEE International. IEEE, 2012, pp 975-978.

[9] H. Rahali, Comportement d'un modèle d'isolateur sous différentes configurations de pollution sous tension alternative $50 \mathrm{~Hz}$, Magister's thesis, Ecole Nationale Polytechnique, Algiers, Algeria, 2011. 